\title{
评述
}

\section{非均相 $\mathrm{TiCl}_{4} / \mathbf{M g C l}_{2}$ 型Ziegler-Natta催化剂催化烯 烃配位聚合机理研究进展}

\author{
牛庆涛, 彭伟, 贺爱华 ${ }^{*}$ \\ 山东省烯烃催化与聚合重点实验室, 橡塑材料与工程教育部重点实验室, 青岛科技大学高分子材料与工程学院, 青岛 266042 \\ *通讯作者, E-mail: ahhe@qust.edu.cn; aihuahe@iccas.ac.cn
}

收稿日期：2019-04-02; 接受日期：2019-05-09; 网络版发表日期：2019-07-11

国家重点基础研究发展计划(编号：2015CB654700，2015CB654706)、国家自然科学基金(编号：51473083)、山东省重大基础研究项目(编号: ZR2017ZA0304)和泰山学者工程项目资助

\begin{abstract}
摘要 非均相 $\mathrm{TiCl}_{4} / \mathrm{MgCl}_{2}$ 型Ziegler-Natta催化剂(负载型Ziegler-Natta催化剂)因其高聚合活性、高立构选择性 及低制备成本, 是目前聚烯烃领域重要的工业催化剂。本文综述了负载型Ziegler-Natta催化剂催化 $\alpha$-烯烃(乙烯、 丙烯)和共轭二烯烃(丁二烯、异戊二烯)配位聚合机理的研究进展, 包括 $\mathrm{TiCl}_{4}$ 在 $\mathrm{MgCl}_{2}$ 表面的吸附、钛的烷基化 与还原、烷基铝的作用、活性中心数目、活性中心价态、活性中心模型、可能活性中心结构及催化机理、给电 子体作用等.最后, 展望了负载型Ziegler-Natta催化剂催化烯烃聚合的机遇与挑战.
\end{abstract}

关键词非均相, 负载型Ziegler-Natta催化剂, $\alpha$-烯烃, 共轭二烯烃, 聚合机理

\section{1 引言}

20 世纪 50 年代, Ziegler等 ${ }^{[1]}$ 和Natta ${ }^{[2]}$ 分别采用 $\mathrm{TiCl}_{4}-\mathrm{AlEt}_{3}$ 催化体系和 $\mathrm{TiCl}_{3}-\mathrm{AlEt}_{2} \mathrm{Cl}$ 催化体系合成高密 度聚乙烯和全同立构聚丙烯，开创了Ziegler-Natta催化 剂合成聚烯烃材料的新时代. 目前全世界范围内每年 采用Ziegler-Natta催化剂和配位聚合方法合成的聚烯 烃材料超过 1 亿吨，约占全部高分子合成材料总量的 $1 / 3^{[3]}$. Ziegler-Natta催化剂主要由过渡金属化合物(主催 化剂)、主族金属烷基化合物(助催化剂)以及给电子

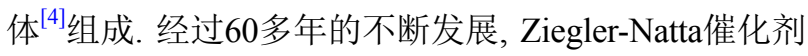
也已经由最初的第一代低活性、低立构规整性的 $\mathrm{TiCl}_{3}$ 型催化剂发展为高活性、高立构规整性、良好的聚合
物颗粒形貌的负载型Ziegler-Natta催化剂. 负载型Ziegler-Natta催化剂生产了全球 $90 \%$ 的聚丙烯和 $50 \%$ 的聚乙 烯, 是目前最为广泛的工业催化剂 ${ }^{[5 \sim 7]}$. 近几十年来, 负 载型Ziegler-Natta催化剂催化的烯烃配位聚合机理的 研究取得了极大的进展, 本文详细综述了 $\mathrm{TiCl}_{4}$ 在 $\mathrm{MgCl}_{2}$ 表面的吸附、烷基化与还原作用机理、活性中心数 目、活性中心价态、活性中心模型、烯烃插入机理、 给电子体的影响、二烯烃配位聚合等方面的研究进展.

\section{$2 \boldsymbol{\alpha}$-烯烃配位聚合机理}

\section{1 $\mathrm{TiCl}_{4}$ 在 $\mathrm{MgCl}_{2}$ 表面的结构}

作为负载型Ziegler-Natta催化剂的载体, $\mathrm{MgCl}_{2}$ 主

引用格式: Niu Q, Peng W, He A. Developments in coordination polymerization mechanism of olefins catalyzed by heterogeneous $\mathrm{TiCl}_{4} / \mathrm{MgCl}_{2}$ type Ziegler-Natta catalyst. Sci Sin Chim, 2019, 49: 1047-1058, doi: 10.1360/SSC-2019-0037 
要是由高缺陷 $\delta$-型 $\mathrm{MgCl}_{2}$ 组成，其存在着 001 面、 104 (或100)面和110面(图1(a)) ${ }^{[8 \sim 11]}$.001面为饱和平面, 104 面和110面分别为5-配位和4-配位的不饱和平面 ${ }^{[12,13]}$. Busico等、Cavallo等、Zakharov等 ${ }^{[14-20]}$ 分别采用密度 泛函理论(DFT)模拟计算发现, $\mathrm{TiCl}_{4}$ 吸附在 $\mathrm{MgCl}_{2}(110)$ 面上是热力学稳定的(图1(b)). Cavallo等 ${ }^{[21]}$ 的阶梯缺陷 (step-defect)模型(图 1(c))指出 $\mathrm{TiCl}_{4}$ 还可吸附于 $\mathrm{MgCl}_{2}$ 的两个104面的阶梯缺陷处的110面上, 很好地解释了 在 $\mathrm{MgCl}_{2}$ 晶体中呈 $90^{\circ}$ 夹角的两晶体层面上都有丙烯 聚合的现象 ${ }^{[22]}$.

$\mathrm{TiCl}_{4}$ 吸附在 $\mathrm{MgCl}_{2}$ 表面上形成络合结构, Brambilla等 ${ }^{[23]}$ 指出在 $\mathrm{TiCl}_{4} / \mathrm{MgCl}_{2}$ 型Ziegler-Natta催化剂中至 少存在三种 $\mathrm{Ti}$ 络合物: (1) 吸附在 $\mathrm{MgCl}_{2}$ (110)面以八面 体结构稳定存在的单核 $\mathrm{TiCl}_{4}$ (图2(a)-Model 1); (2) 吸 附在 $\mathrm{MgCl}_{2}(110)$ 面以四面体结构存在的单核 $\mathrm{TiCl}_{4}$ (图2 (a)-Model 2); (3) 吸附在 $\mathrm{MgCl}_{2}$ (104)面上以团簇态结构 存在的双核 $\mathrm{Ti}_{2} \mathrm{Cl}_{8}$ (图2(a)-Model 3). Busico等 ${ }^{[24]}$ 、Corradini等 ${ }^{[25]}$ 、Cavallo等 ${ }^{[18]}$ 指出吸附在 $\mathrm{MgCl}_{2}$ (104)面上 的双核 $\mathrm{Ti}_{2} \mathrm{Cl}_{8}$ 与烷基铝作用后可以形成等规活性中心; 吸附在 $\mathrm{MgCl}_{2}(110)$ 面上的单核 $\mathrm{TiCl}_{4}$ 在没有添加给电子 体的情况下, 与烷基铝作用形成无规活性中心, 而在添 加给电子体的情况下，与烷基铝作用形成高立构选择 性的活性中心(图2(b)).

催化剂的载 Ti量同样影响着催化剂表面 $\mathrm{TiCl}_{4}$ 的络 合结构和催化剂的催化行为. Koshevoy和Zakharov 等 ${ }^{[26-29]}$ 指出在低Ti含量 $(\leq 0.1 \mathrm{wt} \%)$ 的 $\mathrm{TiCl}_{4} / \mathrm{MgCl}_{2}$ 催化 剂中, $\mathrm{TiCl}_{4}$ 络合结构主要以孤立的单核 $\mathrm{Ti}$ 为主, 其含量 可达30 70 mol\%; 在高 $\mathrm{Ti}$ 含量 $(\geq 1.2 \mathrm{wt} \%)$ 的催化剂中 $\mathrm{TiCl}_{4}$ 络合结构主要以团簇态结构为主 $(60 \sim 90 \mathrm{~mol} \%)$, 此外还含有少量的孤立Ti ( 20 mol\%). Echevskaya
等 ${ }^{[30]}$ 发现在 $\mathrm{TiCl}_{4} / \mathrm{MgCl}_{2}$ 催化剂催化乙烯聚合中，随着 催化剂的载Ti量从 $0.07 \mathrm{wt} \%$ 增加至 $5 \mathrm{wt} \%$, 聚合活性先 增加后快速降低, 形成的聚合物的分子量逐渐增加, 聚 合物的分子量分布逐渐变宽.

\section{2 烷基铝的作用}

(1) 烷基化与还原作用. 烷基铝 $\mathrm{AlR}_{3}$ 与 $\mathrm{TiCl}_{4}$ 通过烷 基化和还原反应形成低价态的 $\mathrm{Ti}$ 活性中心 ${ }^{[15,31 \sim 33]}$, 其 反应过程包括(i) $\mathrm{TiCl}_{4}$ 与 $\mathrm{AlR}_{3}$ 烷基化形成 $\mathrm{TiRCl}_{3}$ 和 $\mathrm{AlR}_{2} \mathrm{Cl}$ ，(ii) $\mathrm{TiRCl}_{3}$ 均裂还原为 $\mathrm{TiCl}_{3}$, (iii) $\mathrm{TiCl}_{3}$ 烷基化 形成 $\mathrm{TiRCl}_{2}$ 和 $\mathrm{AlR}_{2} \mathrm{Cl}$ ，(iv) $\mathrm{TiRCl}_{2}$ 还原和烷基化形成 TiRCl等(图3(a,b)).

Cavallo等 ${ }^{[15]}$ 通过密度泛函理论从能量角度模拟 了烷基化与还原反应过程(图3(c)). 以 $\mathrm{TiCl}_{3}$ 与三乙基铝 $\left(\mathrm{AlEt}_{3}\right)$ 的烷基化与还原反应为例, $\mathrm{AlEt}_{3}$ 通过乙基与 $\mathrm{TiCl}_{3}$ 中的 $\mathrm{Ti}$ 原子配位， $\mathrm{Al}$ 原子与 $\mathrm{Ti}$ 原子通过一个乙基 和Cl桥形成过渡四元环(III), $\mathrm{Al}-\mathrm{Et}$ 键断裂, $\mathrm{AlEt}_{2} \mathrm{Cl}$ 分 子脱离形成活性中心 $\mathrm{TiEtCl}_{2}$.

（2）影响聚合活性. 烷基铝中 R 基团尺寸大小影响 着烷基铝的烷基化和还原的能力，影响着活性中心的

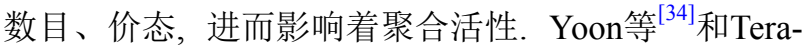
no 等 ${ }^{[35]}$ 在 $\mathrm{TiCl}_{4} / \mathrm{MgCl}_{2}-\mathrm{AlR}_{3}$ 催化丙烯聚合中发现随着 烷基铝中烷基R尺寸增大，聚合活性和活性中心数目 逐渐降低，其由高到低的顺序为三乙基铝(TEA) $>$ 三异 丁基铝(TiBA) $>$ 三己基铝 $(\mathrm{THA})>$ 三辛基铝 $(\mathrm{TOA})>$ 一氯 二乙基铝(DEAC). 烷基 R尺寸变小, 烷基铝与催化剂表 面的 $\mathrm{TiCl}_{4}$ 接触更充分，烷基化与还原能力更强，形成 的活性中心数目变多, 聚合活性也就变大 ${ }^{[33]}$.

（3）调控活性中心的立构规整性. 烷基铝化合物会 吸附、络合在催化剂表面，调控活性中心的立构规整
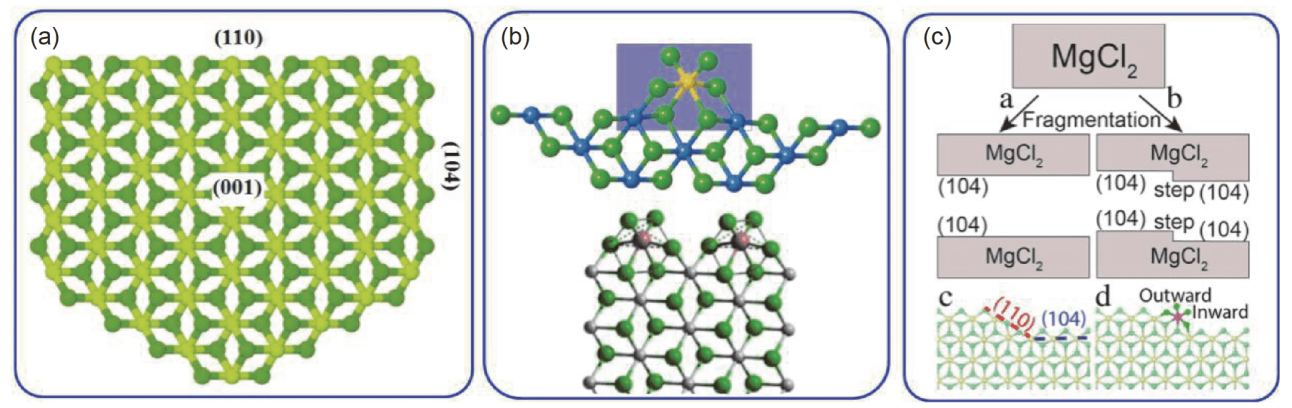

图 1 (a) $\mathrm{MgCl}_{2}$ 晶体表面的(001)、(104)和(110)面 ${ }^{[8]}$; (b) $\mathrm{TiCl}_{4}$ 在 $\mathrm{MgCl}_{2}$ (110)表面吸附 ${ }^{[14,15]}$; (c) 阶梯缺陷模型 ${ }^{[21]}$ (网络版彩图) Figure 1 (a) $\mathrm{MgCl}_{2}$ crystallite showing (001), (104) and (110)-facets [8]; (b) $\mathrm{TiCl}_{4}$ adsorbed on the (110) surface [14,15]; (c) step-defect models [21] (color online). 


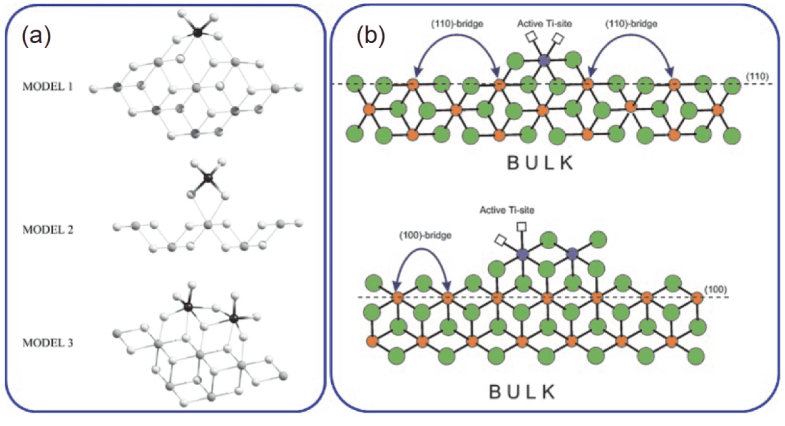

图 2 (a) $\mathrm{TiCl}_{4}$ 吸附模型 ${ }^{[23]}$; (b) $\mathrm{MgCl}_{2}$ 晶体表面活性位点 ${ }^{[18]}$ (网络版彩图)

Figure 2 (a) Models of $\mathrm{TiCl}_{4}$ adsorption [23]; (b) active sites on $\mathrm{MgCl}_{2}$ crystal [18] (color online).

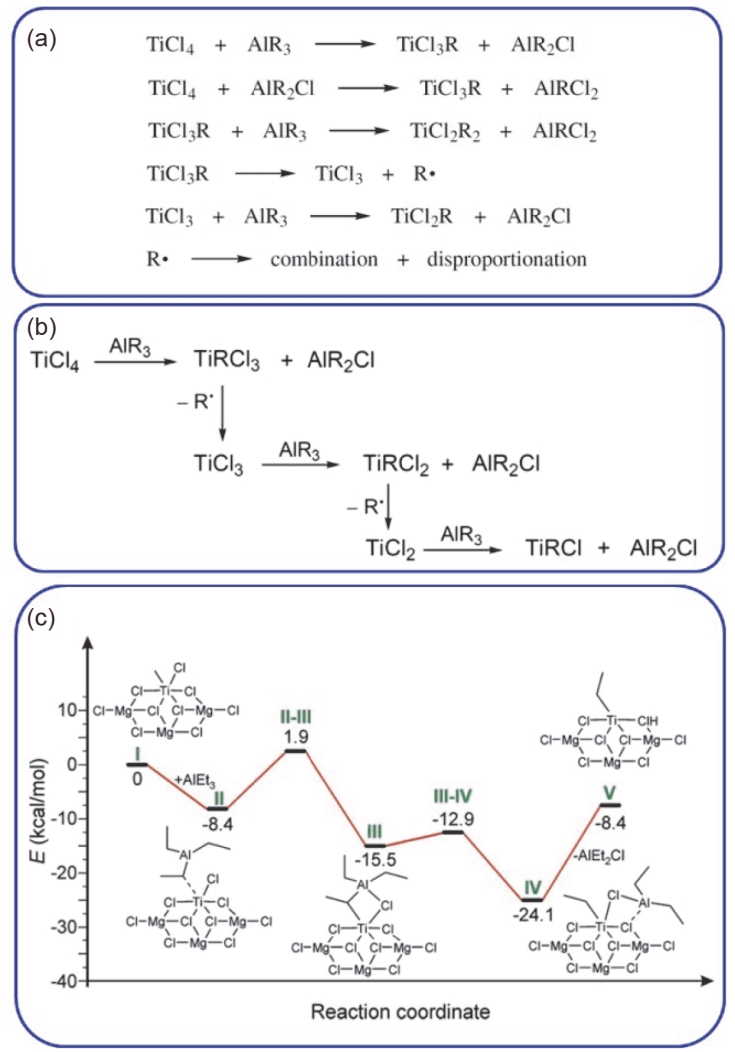

图 3 (a) $\mathrm{TiCl}_{4}$ 与烷基铝之间的复杂反应 ${ }^{[11]}$; (b) 烷基铝与 $\mathrm{Ti}$ 种的烷基化和还原反应 ${ }^{[33]}$; (c) 烷基交换反应能线图 ${ }^{[15]}$ (网络 版彩图)

Figure 3 (a) The complex set of reactions between $\mathrm{TiCl}_{4}$ and alkylaluminum [31]; (b) alkylation and reduction reactions of alkylaluminum with Ti species [33]; (c) energy profile of the direct transalkylation reaction [15] (color online).

性. Potapov等 ${ }^{[36,37]}$ 采用 ${ }^{27} \mathrm{Al}$ NMR MAS发现吸附在催 化剂表面的烷基铝化合物主要为一氯二烷基铝; 范志
强等 ${ }^{[38]}$ 在丙烯聚合中发现一氯二乙基铝 $\mathrm{AlEt}_{2} \mathrm{Cl}$ 会通 过两个 $\mathrm{Cl}$ 桥吸附在活性中心 $\mathrm{Ti}$ 相邻的 $\mathrm{Mg}$ 原子上, 提高 活性中心的立构选择性.

(4) 影响聚合物的分子量及分布. 烷基铝也是一种 链转移剂，会影响着聚合物的分子量及分布. Zakhar$\mathrm{ov}^{[39]}$ 在乙烯聚合中发现提高烷基铝浓度有利于向烷 基铝链转移发生. Yoon 等 ${ }^{[34]}$ 和 Kashiwa $a^{\text {等 }}{ }^{[40]}$ 发现随着 烷基铝中 $\mathrm{R}$ 基团尺寸增加，聚丙烯 $\mathrm{PP}$ 分子量和分布逐 渐增加, 在高温 $\left(50 \sim 100^{\circ} \mathrm{C}\right)$ 聚合时 $\mathrm{PP}$ 分子量分布变窄. 而Terano 等 ${ }^{[35]}$ 发现随着烷基铝中烷基 $\mathrm{R}$ 尺寸增加, 聚 丙烯的分子量逐渐降低, 分子量分布逐渐变窄. 因此, 烷基铝对聚合物的分子量及分布的影响是极其复 杂的.

\section{3 活性中心价态}

$\mathrm{TiCl}_{4}$ 经烷基化和还原反应后转变为低价态的 $\mathrm{Ti}$ 种 $\left(\mathrm{Ti}^{3+}\right.$ 和 $\left.\mathrm{Ti}^{2+}\right)$, 对于不同聚合体系(如丙烯聚合体系、乙 烯聚合体系), 活性中心的价态是不同的. 目前认为 $\mathrm{Ti}^{3+}$ 是丙烯聚合的活性中心价态, $\mathrm{Ti}^{3+}$ 和 $\mathrm{Ti}^{2+}$ 是乙烯聚 合的活性中心价态 ${ }^{[41,42]}$.

聚合时间、烷基铝种类、烷基铝浓度以及活化温 度等聚合因素都会影响催化剂中 Ti的价态及分布( $\left.\mathrm{Ti}^{4+} 、 \mathrm{Ti}^{3+} 、 \mathrm{Ti}^{2+}\right)$. Al-Arifi ${ }^{[41]}$ 和Bresadola等 ${ }^{[42]}$ 在丙烯和 乙烯聚合中发现随着聚合时间增加以及反应温度的提 高, 聚合体系中 $\mathrm{Ti}^{4+}{ }^{4}$ 号量逐渐降低, $\mathrm{Ti}^{3+}$ 含量先增加后 降低, $\mathrm{Ti}^{2+}$ 含量逐渐增加; 提高烷基铝浓度会使 $\mathrm{Ti}^{4+}{ }^{4}$ 含 量降低, 而 $\mathrm{Ti}^{3+}$ 和 $\mathrm{Ti}^{2+}$ 含量增加.

Ternao等 ${ }^{[43,44]}$ 采用 X射线光电子能谱仪(XPS)发现 随着烷基铝中烷基 $\mathrm{R}$ 基团尺寸变小, 催化剂 $\mathrm{Ti}_{2 \mathrm{p} 3 / 2}$ 的键 能(binding energy)降低, 半峰全宽(FWHM)值变宽, Ti 的价态降低.

\section{4 活性中心数目}

20 世纪70年代, Yermakov和Zakharov ${ }^{[45,46]}$ 首次采 用 ${ }^{14} \mathrm{CO}$ 放射同位素标定法(图4(a))测定了丙烯聚合中 的活性中心的数目, 发现在 $\mathrm{TiCl}_{4} /$ 邻苯二甲酸二异丁 酯 $/ \mathrm{MgCl}_{2}-\mathrm{AlEt}_{3} / \mathrm{Si}(\mathrm{OEt})_{4}$ 催化体系中活性中心数目不 足 $1 \%$; 后来, Chien等 ${ }^{[47]} 、$ Tait和Chadwick课题组 ${ }^{[48-51]}$ 运用気醇(ROT) 淬灭法(图4(b)) 测得 $\mathrm{TiCl}_{4} / \mathrm{ID} / \mathrm{MgCl}_{2}$ ( $\mathrm{ID}=$ 苯甲酸乙酯、邻苯二甲酸酯、二醚) 催化丙烯聚 合中活性中心数目分别为 $7 \% \sim 30 \% 、 21 \% \sim 22 \% 、 2 \%$ 

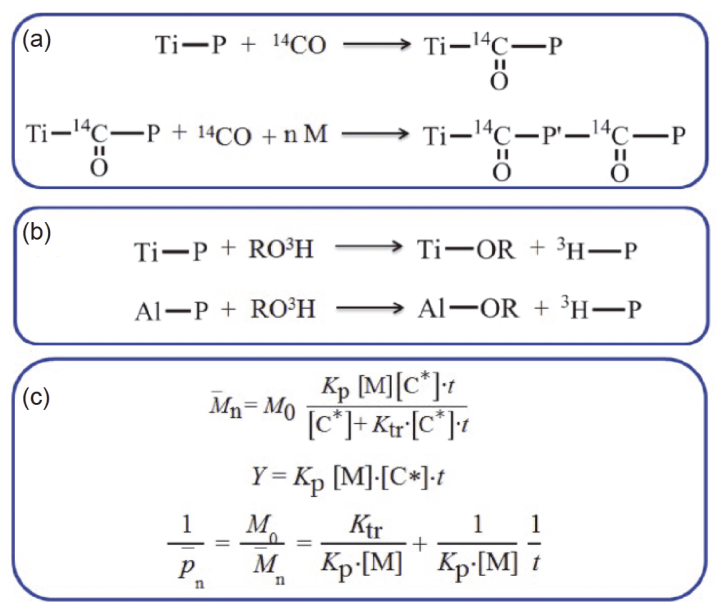

$\begin{array}{rl}\text { (d) } & \mathrm{Ti}-\mathrm{P}+\mathrm{RCOCl} \longrightarrow \mathrm{Ti}-\mathrm{Cl}+\mathrm{R}-\stackrel{\mathrm{O}}{\mathrm{C}}-\mathrm{P} \\ \mathrm{Al}-\mathrm{P}+\mathrm{RCOCl} \longrightarrow \mathrm{O} & \mathrm{Al}-\mathrm{Cl}+\mathrm{R}-\mathrm{C}-\mathrm{C}\end{array}$

图 4 (a) ${ }^{14} \mathrm{CO}$ 放射标定法, (b) 気醇法, (c) stpopped-flow法, (d) 酰氯淬灭法的方程(网络版彩图)

Figure 4 The equation of (a) ${ }^{14} \mathrm{CO}$ radiotagging method, (b) tritiated ethanol method, (c) stopped-flow method, (d) acyl chloride quenching method (color online).

$8 \%$. Terano 等 ${ }^{[52]}$ 采用 stopped-flow方法(图4(c))测得丙 烯聚合中活性中心数目为 $5.2 \sim 7.5 \mathrm{~mol} \%$, 活性中心数 目随着烷基铝浓度的增大而增大，随着烷基铝中烷基 $\mathrm{R}$ 基团尺寸减小而增大. ${ }^{14} \mathrm{CO}$ 与活性链的接触反应需 要较长时间, 而且只能与少部分的活性中心反应, stopped-flow法只能用于测定聚合初期的活性中心 数目.

最近，范志强等 ${ }^{[33-58]}$ 基于酰氯与活性链的反应建 立了一系列以酰氯为淬灭剂测定活性中心数目的方法 (图4(d)), 其测得 $\alpha$-烯烃 (乙烯、丙烯、1-辛烯、1-已烯) 聚合中的活性中心数目为 $1.3 \%$ \% . $8 \%$ ，通过抑制副反 应，优化淬灭条件测得丙烯聚合中的活性中心数目为

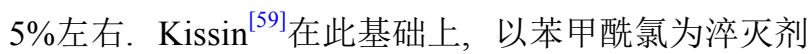
测得 $\mathrm{Ti}$ 基型催化剂催化乙烯和丙烯聚合的活性中心数 目为 $1 \% \sim 3 \%$.

\section{5 活性中心模型及立构选择性}

$\alpha$-烯烃经配位聚合可形成低等规、高等规和间规 聚合物，不同立构选择性的活性中心决定了聚合物的 立构规整性.

(1) 双金属模型. van Looy、Keii等 ${ }^{[60-62]}$ 最早提出
了等规活性中心为Ti-Al双金属活性中心模型(图5(a)), 认为在 $\mathrm{TiCl}_{3}$ 型催化剂催化丙烯聚合中形成的无规活 性中心是具有一个或两个空位的单金属 $\mathrm{Ti}$ ，其与烷基 铝 $\left(\mathrm{AlR}_{3}\right.$ 或 $\left.\mathrm{AlR}_{2} \mathrm{Cl}\right)$ 发生可逆络合后可转变为双金属 Ti$\mathrm{Al}$ 等规活性中心. $\mathrm{Doi}^{[63]}$ 和徐君庭等 ${ }^{[64]}$ 认为 $\mathrm{AlR}_{3}$ 与 $\mathrm{Ti}$ 的 可逆转换配位可形成等规、间同的双金属活性中心， 该模型可解释分子链中全同和间同嵌段共存的现象 (图5(b)).

(2) 单金属模型. 最近几十年, Busico 等 ${ }^{[6568]}$ 提出 了“three-sites”单金属模型(图6(a)), 认为活性中心中存 在着三种具有不同立构选择性的可相互转变的高等 规、低等规以及间规单金属活性中心. 活性中心中 $\mathrm{L}_{1}$ 和 $\mathrm{L}_{2}$ 处配体的空间位阻的大小直接决定了活性中心的 立构选择性的高低. 单活性中心模型得到国内外学者 的进一步认可, Terano等 ${ }^{[6971]}$ 指出烷基铝通过不断与 具有不同化学环境的活性中心前驱体 $\mathrm{TiCl}_{4} / \mathrm{MgCl}_{2}$ 活 化、吸附、络合形成具有不同立构选择性的活性中心 (图6(b)). 活性中心周围的化学环境如空位的数目, 悬 挂的 $\mathrm{Cl}$ 原子个数, 活性 $\mathrm{Ti}$ 原子在催化剂基体表面的暴 露程度以及 $\mathrm{L}_{1} 、 \mathrm{~L}_{2}$ 配体位置上的取代基决定了活性中 心立构选择性的高低. Potapov及范志强等 ${ }^{[36-38]}$ 进一步

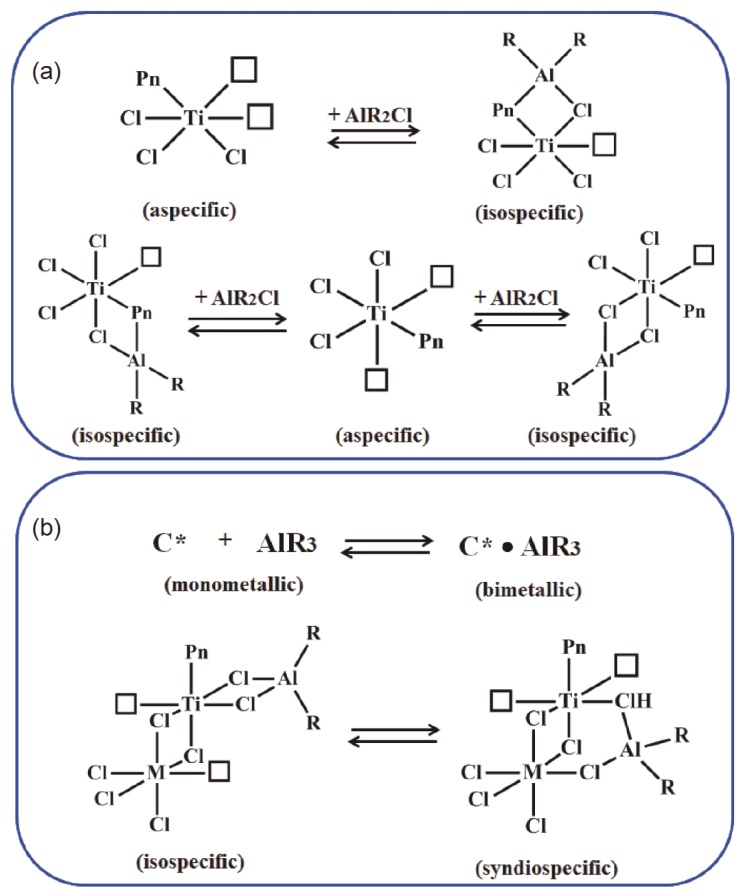

图 5 双金属模型 ${ }^{[62]}$ (网络版彩图)

Figure 5 Bimetallic active centers models [62] (color online). 

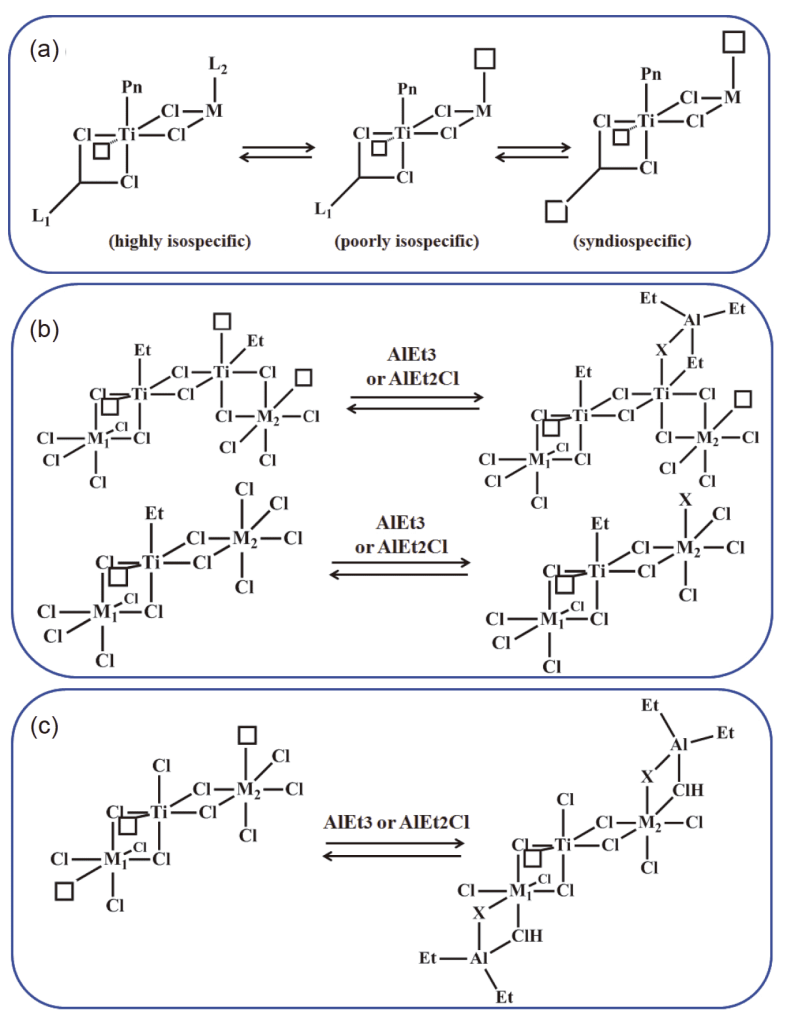

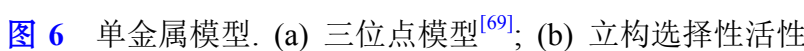
中心形成转变机理模型 ${ }^{[69]}$; (c) $\mathrm{AlEt}_{2} \mathrm{Cl}$ 机理模型 ${ }^{[38]}$ (网络版 彩图)

Figure 6 Monometallic active centers models. (a) Three-sites models [69]; (b) mechanism models of formation and transformation of stereospecific active sites [69]; (c) mechanism models of $\mathrm{AlEt}_{2} \mathrm{Cl}$ [38] (color online).

指出吸附在催化剂表面的烷基铝化合物主要为一氯二 烷基铝 $\mathrm{AlR}_{2} \mathrm{Cl}$ ，其既可吸附在 $\mathrm{Ti}$ 原子上使活性中心失 活，又可吸附在 $\mathrm{Ti}$ 相邻的 $\mathrm{Mg}$ 原子上提高活性中心的等 规度, 还可与没有活性的二价钛络合, 形成新的活性中 心(图6(c)).

(3) 增长机理. 基于聚烯烃在 $\alpha-\mathrm{TiCl}_{3}$ 晶体表面的电 子显微镜照片和 $\mathrm{TiCl}_{3}$ 结构分析, Cossee和 $\mathrm{Arlman}^{[72 \sim 75]}$ 在1964年提出了烯烃配位插入增长机理，其指出催化 剂的活性位点主要位于 $\mathrm{TiCl}_{3}$ 晶体中不饱和配位的 边、角位置, 该位置的 $\mathrm{Ti}$ 原子与一个空位和 5 个 $\mathrm{Cl}$ 原子 相连, 其中一个 $\mathrm{Cl}$ 原子为自由 $\mathrm{Cl}$, 可被烷基铝中的烷基 $\mathrm{R}$ 取代, 形成Ti-R键， $\alpha$-烯烃如乙烯单体通过空位与活 性中心金属Ti原子配位, 络合形成过渡四元环, 然后插 入到 Ti-R键中, 空出一个空位, 供下一个单体配位增长 (图7).

在烯烃聚合中, 活性增长链会与单体、氢气、烷

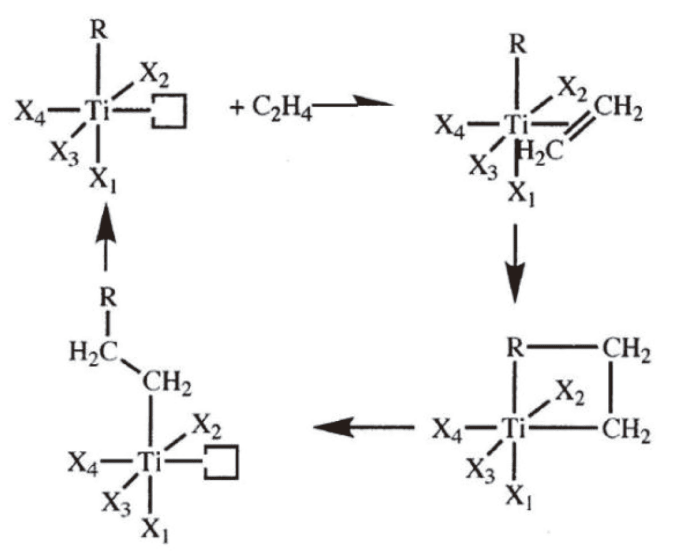

图 7 Cossee 和Arlman提出的 $\alpha$-烯烃配位聚合模型 ${ }^{[72 ~ 75] ~}$

Figure $7 \alpha$-olefin coordination polymerization models proposed by Cossee and Arlman [72-75].

基铝等进行链转移反应，以乙烯单体为例，其链转移 反应如下(P代表聚合物链)(Eq.-1 Eq.-3). 在这三种链 转移反应中, 向烷基铝 $\mathrm{AlR}_{3}$ 链转移主要发生于聚合初 期，在聚合物链末端形成 $\mathrm{AlR}_{2}$ 基团，随着聚合时间增 加, 向烷基铝 $\mathrm{AlR}_{3}$ 链转移反应程度逐渐降低, 向单体 链转移反应程度逐渐增加; 在添加氢气的情况下, 链 转移以向 $\mathrm{H}_{2}$ 链转移反应为主, 因此氢气常用作链转移 剂调节聚合物的分子量及分布; 形成的 Ti-H会快速地 与单体反应转变为新的活性中心 ${ }^{[39,76]}$.

$$
\begin{aligned}
\mathrm{Ti}-\mathrm{CH}_{2} \mathrm{CH}_{2}-\mathrm{P} & +\mathrm{CH}_{2}=\mathrm{CH}_{2} \longrightarrow \text { (Eq.-1) } \\
\mathrm{Ti}-\mathrm{CH}_{2} \mathrm{CH}_{3} & +\mathrm{CH}_{2}=\mathrm{CH}-\mathrm{P} \\
\mathrm{Ti}-\mathrm{CH}_{2} \mathrm{CH}_{2}-\mathrm{P} & +\mathrm{H}_{2} \longrightarrow \text { (Eq.-2) } \\
\mathrm{Ti}-\mathrm{H} & +\mathrm{CH}_{3} \mathrm{CH}_{2}-\mathrm{P} \\
\mathrm{Ti}-\mathrm{CH}_{2} \mathrm{CH}_{2}-\mathrm{P} & +\mathrm{AlR}_{3} \longrightarrow \text { (Eq.-3) } \\
\mathrm{Ti}-\mathrm{R} & +\mathrm{R}_{2} \mathrm{Al}^{-\mathrm{CH}_{3} \mathrm{CH}_{2}} \longrightarrow \mathrm{P}
\end{aligned}
$$

烯烃配位聚合链终止比较困难, 经过长时间, 可能 会发生向分子链内的 $\beta-H$ 转移而自身终止. 水、醇、 酸、胺等含活性氢的化合物是配位聚合常用的终止 剂[77].

\section{6 给电子体对立构选择性的影响}

在 $\mathrm{TiCl}_{4} / \mathrm{MgCl}_{2} / \mathrm{Di}-\mathrm{AlR}_{3} / \mathrm{De}$ 型的Ziegler-Natta催化 剂中, 添加的内、外给电子体会明显地影响着催化剂 的催化性能. 在催化剂制备时加入的内给电子体会吸 附在 $\mathrm{MgCl}_{2}$ 晶体表面, 改变 $\mathrm{MgCl}_{2}$ 晶体表面的结 
构 $^{[78 \sim 81]}$. Cavallo等 ${ }^{[17]}$ 指出内给电子会通过移除 $\mathrm{MgCl}_{2}$ 晶体两 104 面夹角处的 $\mathrm{MgCl}_{2}$ 单元, 形成多缺陷的 110 面(图8(a)), 使 $\mathrm{MgCl}_{2}$ 表面结构重新排列, 内给电子体 吸附在 $\mathrm{TiCl}_{4}$ 活性中心周围相邻两端的 104 或 110 面上 (1,3-二醚、琥珀酸酯中两配位氧原子间距长，配位在 104面上; 而烷基硅氧烷中的两个氧原子间距短, 吸附 在110面上), 改变催化剂表面的空间位阻和络合稳定 性, 提高活性中心的立构选择性 ${ }^{[17,82]}$. 当 $\mathrm{TiCl}_{4} / \mathrm{MgCl}_{2} /$ $\mathrm{Di}$ 催化剂与烷基铝 $\mathrm{AlR}_{3}$ 接触时, 烷基铝与内给电子体 发生络合或烷基反应，使吸附在催化剂表面的内给电 子体脱离, 在无外给电子体情况下, 催化剂的立构选 择性会降低，而在添加的外给电子体情况下，外给电 子体会部分取代内给电子体，形成更高立构选择性的 活性中心 ${ }^{[83 ~ 87]}$.

Kakugo等 ${ }^{[88}$ 指出在聚合过程中加入的外给电子 体可以有效地降低聚合体系中的无规位点、增加等规 位点, 提高聚合物的等规度. 范志强等 ${ }^{[89]}$ 指出外给电 子体(烷基硅氧 $\mathrm{R}_{1} \mathrm{R}_{2} \mathrm{Si}(\mathrm{OMe})_{2}$ ) 既可吸附在活性中心 $\mathrm{Ti}$ 原子周围相邻的两个 $\mathrm{Mg}$ 原子上, 提高活性中心的立构 选择性，还可以同时吸附在活性中心的 Ti原子和相邻 的 Mg原子上, 使活性中心失活, 降低聚合活性(图8(b)).

Busico和Cipullo等 ${ }^{[90]}$ 在最新研究成果中指出, 外 给电子体和烷基铝化合物 $\left(\mathrm{AlEt}_{2} \mathrm{Cl}\right)$ 可协同地吸附在活 性中心 $\mathrm{Ti}$ 原子相邻的 $\mathrm{Mg}$ 原子上, 即烷基铝和外给电子 体分别吸附在 $\mathrm{Ti}$ 原子相邻的和次相邻的 $\mathrm{Mg}$ 原子上, 改 变催化剂表面的覆盖度和化学位阻, 形成更稳定的吸 附状态(图8(c)).

\section{3 二烯烃配位聚合机理}

目前关于过渡金属催化剂催化二烯烃配位聚合机 理研究多集中在均相催化剂体系, 而非均相催化剂催 化二烯烃配位聚合的催化机理研究极少.

\section{1 均相过渡金属催化剂催化二烯烃配位聚合 机理}

均相过渡金属催化剂催化二烯烃(丁二烯或异戊 二烯)聚合是一种配位插入聚合 ${ }^{[91]}$. 1966年, Cosse $e^{[92]}$ 和Arlman ${ }^{[93]}$ 最早提出 $\sigma$-烯丙基插入聚合机理, 丁烯基 以 $\eta^{1}-\sigma$ 形式存在; 随后Taube 等 ${ }^{[94-96]}$ 通过DFT模拟计算 提出了更有信服力的 $\pi$-烯丙基插入机理, 丁烯基以 $\eta^{3}-\pi$
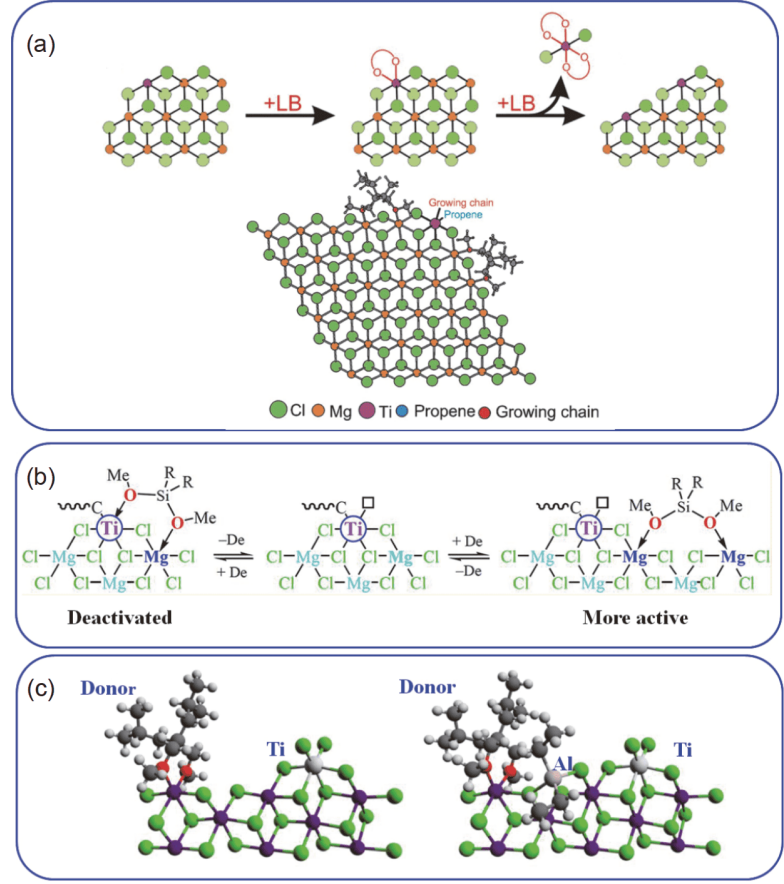

图 8 给电子体作用模型. (a) $\mathrm{MgCl}_{2}$ 重构和给电子体在 $\mathrm{Ti}$ 两 端配位 ${ }^{[17]} ;$ (b) 给电子体与 $\mathrm{MgCl}_{2}$ (110)面上的 Ti配位 ${ }^{[89]}$; (c) DFT模拟给电子体和 $\mathrm{AlEt}_{2} \mathrm{Cl}$ 在 $\mathrm{MgCl}_{2}$ (110)面协同配位 ${ }^{[90]}$ (网 络版彩图)

Figure 8 Donor models. (a) Reconstruction of $\mathrm{MgCl}_{2}$ and coordination of donor on both sides of Ti-species [17]; (b) coordination of donor molecule on a mono-Ti active site located on the (110) lateral cut of $\mathrm{MgCl}_{2}$ crystallite [89]; (c) DFT model of adjacent $\mathrm{TiCl}_{4}$ and donor coadsorption on a $\mathrm{MgCl}_{2}(110)$ edge and same as panel (a) after the adsorption of an $\mathrm{AlEt}_{2} \mathrm{Cl}$ molecule [90] (color online).

形式存在. $\pi$-烯丙基插入机理主要包括: 单体的插入、 烯丙基syn-anti 异构化、cis-trans 选择性控制等过 程 ${ }^{[97,98]}$.

(1) 单体插入: 二烯烃单体与金属中心配位存在单 齿 $\left(\eta^{2}\right)$ 和二齿 $\left(\eta^{4}\right)$ 配位, 双齿配位的单体又存在 $s$ - cis ( $s$ 顺式)和s-trans ( $s$-反式)两种构象. 单体以 $\eta^{4}$-cis构象配 位形成anti- $\eta^{3}$-烯丙基，得到 $c i s-1,4-$ 结构，以 $\eta^{4}$-trans 构 象配位形成 $s y n-\eta^{3}$-烯丙基, 得到 trans-1,4-结构(图9, 以 丁二烯为例).

（2）anti-syn异构化: 在烯丙基镍(II)催化丁二烯聚 合过程中，烯丙基 syn-anti异构化是生成trans-1,4结构 必不可缺少的过程 ${ }^{[99]}$. 通过anti-syn 异构化, anti- $\eta^{3}$-烯 丙基转化为热力学上更稳定的 $s y n-\eta^{3}$-烯丙基, 得到 trans-1,4结构的聚合物, 其异构化具体过程为: anti- $\eta^{3}-$ $\pi$-烯丙基通过 $\pi \rightarrow \sigma$ 或 $\eta^{3} \rightarrow \eta^{1}$ 转化为 $\eta^{1}-\sigma-\mathrm{C}_{3}$ 丁烯基, 丁烯 


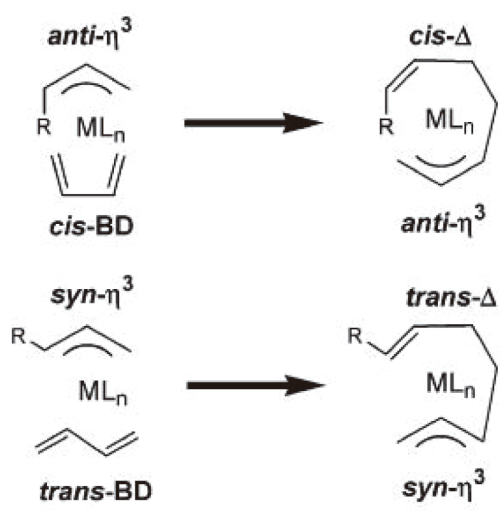

图 $9 A n t i$-cis 和 syn-trans 对应关系 ${ }^{[97]}$

Figure 9 Anti-cis and syn-trans correlation [97].

基中的乙烯基绕着 $\mathrm{C}_{2}-\mathrm{C}_{3}$ 单键进行 outward或inward旋 转得到 $\eta^{1}-\sigma-\mathrm{C}_{3}$ 丁烯基中间体，再通过 $\sigma \rightarrow \pi$ 或 $\eta^{1} \rightarrow \eta^{3}$ 转 化为热力学更稳定的 $s y n-\eta^{3}$ - $\pi$-烯丙基.

(3) cis-trans 选择性控制与anti-syn 异构化: 从动力 学的角度上讲, 聚合物 cis-trans 结构的选择性是由anti和syn-丁烯基的反应活性(与单体的插入反应)和antisyn 异构化速率的大小决定的. 当anti-syn异构化位垒远 低于单体插入位垒时，低反应活性的anti-丁烯基络合 物转变为高反应活性的syn-丁烯基络合物，进而得到 trans-1,4结构单元的聚合物; 当anti-syn 异构化位垒明 显高于单体插入位垒时, anti-syn 异构化速率要远低于 单体插入速率，形成顺式-1,4-结构单元聚合物. To$\operatorname{bisch}^{[97]}$ 指出催化体系的化学环境(如催化剂空间位阻 大小)、聚合温度等直接决定了异构化位阻与单体插 入位阻之间的大小, 进而决定了催化剂的cis-trans选 择性.

\section{2 非均相 $\mathrm{TiCl}_{4} / \mathrm{MgCl}_{2}$ 型Ziegler-Natta催化剂催 化丁二烯/异戍二烯聚合}

非均相 $\mathrm{TiCl}_{4} / \mathrm{MgCl}_{2}$ 型Ziegler-Natta催化剂催化丁 二烯、异戊二烯均聚合及共聚合，得到高trans $-1,4$ 结 构的聚合物，该聚合物具有优异的耐疲劳、耐屈挠、 低压缩生热等性能，是制备绿色高性能轮胎的理想胶 料 ${ }^{[100 ~ 106]}$.

\subsection{1 聚合行为}

黄宝琛等 ${ }^{[100,101]}$ 首次采用非均相 $\mathrm{TiCl}_{4} / \mathrm{MgCl}_{2}$ - $\mathrm{Al}(\mathrm{i}$ $\mathrm{Bu})_{3}$ 催化体系催化异戊二烯和丁二烯均聚, 合成了高 trans $-1,4$ 结构的聚异戊二烯(TPI)和聚丁二烯(TPB). 贺 爱华等 ${ }^{[105,107 ~ 115]}$ 在 $\mathrm{TiCl}_{4} / \mathrm{MgCl}_{2}-\mathrm{Al}(\mathrm{i}-\mathrm{Bu})_{3}$ 催化丁二烯异戊二烯共聚合中系统地研究了单体配比、烷基铝浓 度、聚合温度等因素对共聚合的影响，发现随温度、 烷基铝浓度 $(\mathrm{Al} / \mathrm{Ti})$ 、催化剂载 $\mathrm{Ti}$ 量的增加，聚合活性 先逐渐增加后缓慢降低 ${ }^{[102,103]}$; 聚合速率对单体浓度 $[\mathrm{M}]$ 和催化剂浓度 $[\mathrm{Ti}]$ 的反应级数为一级, 聚合速率方 程为 $R=K_{\mathrm{p}} \cdot f \cdot[\mathrm{Ti}] \cdot[\mathrm{M}]^{[107,108]}$. 丁二烯单体的聚合速率远 大于异戊二烯单体的 ${ }^{[109,110]}$, 丁二烯单体竞聚率 $r_{\mathrm{Bd}}$ 为 $5.28 \sim 5.20$, 异戊二烯单体竞聚率 $r_{\mathrm{Ip}}$ 为 $0.20 \sim 0.17$; 聚合 条件对共聚物的微观结构影响不大，其中丁二烯单元 反式-1,4结构含量大于 $98 \mathrm{~mol} \%$, 异成二烯单元反式1,4 结构含量大于 $90 \mathrm{~mol} \%$ ，共聚物的序列分布服从一

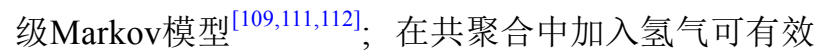
地调节聚合物的分子量及分布 ${ }^{[113,114]}$.

\subsubsection{Trans-1,4聚合机理}

贺爱华等 ${ }^{[116,117]}$ 通过异戊二烯和丁二烯共聚合动 力学研究发现, 在聚合初期得到的共聚物是一种含有 高 $c i s-1,4$ 结构含量的cis-1,4/trans-1,4 (42\%/56\%)混合 结构, 随着聚合时间的增加, 共聚物中 cis-1,4结构含量 逐渐降低, trans $-1,4$ 结构含量逐渐增加并最终成为共 聚物的主结构 $(96 \mathrm{mo} \%)$. 逐步等温结晶分级表明共聚 物主要是由两大类级份组成: 以 cis-1,4结构为主的级 份和以 trans-1,4结构为主的级份, 前者由低trans-1,4立 构选择性的活性中心形成，后者由高trans-1,4立构选 择性的活性中心形成. 由此，贺爱华等提出了非均相 $\mathrm{TiCl}_{4} / \mathrm{MgCl}_{2}$ 型Ziegler-Natta催化剂催化异戊二烯-丁二 烯trans-1,4定向聚合的机理模型(图10): 烷基铝与活性 中心前驱体 $\mathrm{TiCl}_{4}$ 通过烷基化和还原反应首先形成低 空间位阻的活性中心 $C^{*}-2$, 该活性中心具有低的trans1,4 立构选择性, 允许丁二烯和异戊二烯单体以 $s$ - cis 构 象形式与活性中心 $\mathrm{C}^{*}-2$ 的中心金属 $\mathrm{Ti}$ 原子配位，形成 anti-型的 $\pi$-烯丙基，进而得到 $c i s-1,4$ 序列结构; anti-型 的 $\pi$-烯丙基在热力学上是不稳定的, 其通过anti-syn 异 构化转变为热力学上稳定的 $s y n$-型 $\pi$-烯丙基, 进而得 到trans-1,4序列结构, 因此在聚合初期形成的聚合物 是一种含有高 $c i s-1,4$ 结构的 $c i s-1,4 /$ trans $-1,4$ 混合结构; 烷基铝与 $\mathrm{TiCl}_{4}$ 反应产物一氯二烷基铝 $\left(\mathrm{Al}(\mathrm{i}-\mathrm{Bu})_{2} \mathrm{Cl}\right)$ 会 通过两个 $\mathrm{Cl}$ 桥与活性中心 $\mathrm{C}^{*}-2$ 周围相邻的 $\mathrm{Mg}$ 原子发 生可逆吸附、络合，将活性中心 $C^{*}-2$ 转化为高空间位 


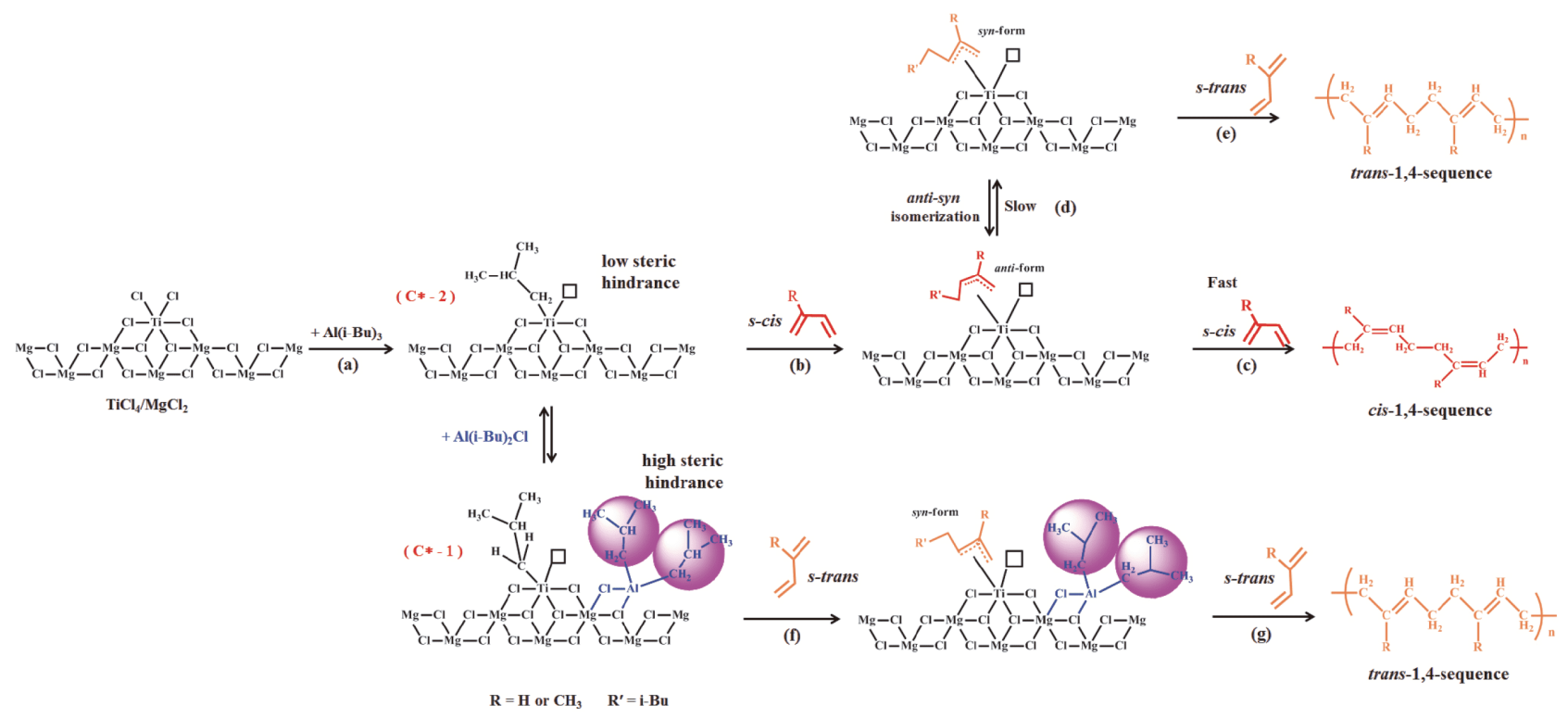

图 $10 \mathrm{TiCl}_{4} / \mathrm{MgCl}_{2}-\mathrm{Al}(\mathrm{i}-\mathrm{Bu})_{3}$ 催化共轭二烯烃聚合机理以及可能的活性位点 ${ }^{[117]}$ (网络版彩图)

Figure 10 Proposed active center sites and polymerization mechanism of conjugated dienes with $\mathrm{TiCl}_{4} / \mathrm{MgCl}_{2}-\mathrm{Al}(\mathrm{i}-\mathrm{Bu})_{3}$ system [117] (color online).

阻的活性中心 $\mathrm{C}^{*}-1$, 该活性中心具有高的trans-1,4立 构选择性，允许异戊二烯和丁二烯单体以s-trans构象 形式与活性中心 $\mathrm{C}^{*}-1$ 的中心金属 $\mathrm{Ti}$ 原子配位, 形成 $s y n$-型 $\pi$-烯丙基, 进而得到trans-1,4序列结构; 随着活 性中心 $C^{*}-2$ 逐渐转变为活性中心 $C^{*}-1, C^{*}-1$ 最终成为 聚合体系的主活性中心, 形成的聚合物也最终以高 trans-1,4结构为主.

\section{4 总结与展望}

本文从非均相 $\mathrm{TiCl}_{4} / \mathrm{MgCl}_{2}$ 型Ziegler-Natta催化剂 催化 $\alpha$-单烯烃和共轭二烯烃配位聚合两个方面总结介 绍了近几十年的烯烃配位聚合机理的研究进展, 涉及 $\mathrm{TiCl}_{4}$ 在催化剂表面络合吸附、烷基化与还原反应形
成活性中心、活性中心的价态及数目、活性中心结构 和空间环境对立构选择性的改变、烯烃聚合增长机理 等. 由于 $\mathrm{TiCl}_{4} / \mathrm{MgCl}_{2}$ 型Ziegler-Natta催化剂催化烯烃聚 合是一个极度复杂的体系, 尚待解决的问题有: (1) 活 性中心前驱体在催化剂表面具体位点及周围化学环 境; (2) 活性中心的化学结构类型及其对催化剂立构选 择性、聚合活性及动力学、聚合物分子量等的影响; (3) 活性中心的形成、转变等演变过程. 因此仍需继续 加强非均相 $\mathrm{TiCl}_{4} / \mathrm{MgCl}_{2}$ 型Ziegler-Natta催化剂催化共 轭二烯烃配位聚合的机理的研究. 相比其他催化剂如 茂金属催化剂和单活性中心催化剂, 均相 $\mathrm{TiCl}_{4} / \mathrm{MgCl}_{2}$ 型Ziegler-Natta催化剂具有高活性、高立构选择性的 优势, 在今后很长一段时间内仍然是制备聚烯烃材料 的主体催化剂.

\section{参考文献}

1 Ziegler K, Holzkamp E, Breil H, Martin H. Angew Chem, 1955, 67: 541-547

2 Natta G. J Polym Sci, 1955, 16: 143-154

3 Busico V. Adv Polym Sci, 2013, 257: 37-57

4 Boor J Jr. Ziegler-Natta Catalysts and Polymerizations. New York: Academic Press, 1979. 33-35

5 Soga K. Prog Polym Sci, 1997, 22: 1503-1546

6 Huang J. Prog Polym Sci, 1995, 20: 459-526 
7 Chang M, Liu X, Nelson P, Munzing G, Gegan T, Kissin Y. J Catal, 2006, 239: 347-353

8 Kuklin MS, Bazhenov AS, Denifl P, Leinonen T, Linnolahti M, Pakkanen TA. Surf Sci, 2015, 635: 5-10

9 Bazhenov A, Linnolahti M, Pakkanen TA, Denifl P, Leinonen T. J Phys Chem C, 2014, 118: 4791-4796

10 Giannini U, Giunchi G, Albizzati E, Barbe PC. Frontiers in Polymerization Catalysis. NATO Advanced Research Workshop Technical Report, 1987

11 Mori H, Sawada M, Higuchi T, Hasebe K, Otsuka N, Terano M. Macromol Rapid Commun, 1999, 20: 245-250

12 Stukalov DV, Zakharov VA, Potapov AG, Bukatov GD. J Catal, 2009, 266: 39-49

13 Busico V, Causà M, Cipullo R, Credendino R, Cutillo F, Friederichs N, Lamanna R, Segre A, Van Axel Castelli V. J Phys Chem C, 2008, 112: 1081-1089

14 D’Amore M, Credendino R, Budzelaar PHM, Causá M, Busico V. J Catal, 2012, 286: 103-110

Bahri-Laleh N, Correa A, Mehdipour-Ataei S, Arabi H, Haghighi MN, Zohuri G, Cavallo L. Macromolecules, 2011, 44: 778-783

Capone F, Rongo L, D’Amore M, Budzelaar PHM, Busico V. J Phys Chem C, 2013, 117: 24345-24353

Monaco G, Toto M, Guerra G, Corradini P, Cavallo L. Macromolecules, 2000, 33: 8953-8962

Correa A, Credendino R, Pater JTM, Morini G, Cavallo L. Macromolecules, 2012, 45: 3695-3701

Correa A, Piemontesi F, Morini G, Cavallo L. Macromolecules, 2007, 40: 9181-9189

Stukalov DV, Zilberberg IL, Zakharov VA. Macromolecules, 2009, 42: 8165-8171

Credendino R, Liguori D, Fan Z, Morini G, Cavallo L. ACS Catal, 2015, 5: 5431-5435

Andoni A, Chadwick J, Niemantsverdriet H, Thune P. J Catal, 2008, 257: 81-86

Brambilla L, Zerbi G, Piemontesi F, Nascetti S, Morini G. J Mol Catal A-Chem, 2007, 263: 103-111

Busico V, Corradini P, De Martino L, Proto A, Savino V, Albizzati E. Makromol Chem, 1985, 186: 1279-1288

Corradini P, Barone U, Fusco R, Guerra G. Gazz Chim Ital, 1983, 113:601-607

Koshevoy EI, Mikenas TB, Zakharov VA, Volodin AM, Kenzhin RM. Catal Commun, 2014, 48: 38-40

Koshevoy EI, Mikenas TB, Zakharov VA, Shubin AA, Barabanov AA. J Phys Chem C, 2016, 120: 1121-1129

Zakharov VA, Makhtarulin SI, Poluboyarov VA, Anufrienko VF. Makromol Chem, 1984, 185: 1781-1793

Sergeev SA, Poluboyarov VA, Zakharov VA, Anufrienko VF, Bukatov GD. Makromol Chem, 1986, 187: 243-255

Echevskaya LG, Matsko MA, Mikenas TB, Nikitin VE, Zakharov VA. J Appl Polym Sci, 2006, 102: 5436-5442

Odian G. Principles of Polymerization. New Jersey: John Wiley \& Sons, Inc., 2004. 645

Kuran W. Principles of Coordination Polymerization. Chichester: John Wiley \& Sons Ltd, 2001. 56-57

Zhang B, Dong Q, Fu Z, Fan Z. Polymer, 2014, 55: 4865-4872

Zhang HX, Lee YJ, Park JR, Lee DH, Yoon KB. J Appl Polym Sci, 2011, 120: 101-108

Mori H, Hasebe K, Terano M. Polymer, 1999, 40: 1389-1394

Potapov AG, Terskikh VV, Zakharov VA, Bukatov GD. J Mol Catal A-Chem, 1999, 145: 147-152

Potapov AG, Terskikh VV, Bukatov GD, Zakharov VA. J Mol Catal A-Chem, 2000, 158: 457-460

Hu J, Han B, Shen X, Fu Z, Fan Z. Chin J Polym Sci, 2013, 31: 583-590

Nikolaeva MI, Mikenas TB, Matsko MA, Echevskaya LG, Zakharov VA. J Appl Polym Sci, 2011, 122: 3092-3101

Kojoh S, Kioka M, Kashiwa N. Eur Polym J, 1999, 35: 751-755

Al-Arifi ASN. J Appl Polym Sci, 2004, 93: 56-62

Fregonese D, Mortara S, Bresadola S. J Mol Catal A-Chem, 2001, 172: 89-95

Hasebe K, Mori H, Terano M. J Mol Catal A-Chem, 1997, 124: L1-L3

Mori H, Hasebe K, Terano M. J Mol Catal A-Chem, 1999, 140: 165-172

Yermakov YI, Zakharov VA. Coordination Polymerization. Chien JCW, ed. New York: Academic, 1975. 91-133

Bukatov GD, Zakharov VA. Macromol Chem Phys, 2001, 202: 2003-2009

Chien JCW, Kuo CI. J Polym Sci Polym Chem Ed, 1985, 23: 731-760

Tait PJT, Zohuri GH, Kells AM, McKenzie ID. Ziegler Catalysts. Recent Scientific Innovations and Technological Improvements. Fink G, Mülhaupt R, Brintzinger HH, eds. Berlin: Springer-Verlag, 1995. 343-362

Yaluma AK, Tait PJT, Chadwick JC. J Polym Sci A Polym Chem, 2006, 44: 1635-1647 
Marques MM, Tait PJT, Mejzlk J, Dias AR. J Polym Sci A Polym Chem, 1998, 36: 573-585

Mejzlik J, Lesna M, Majer J. Makromol Chem, 1983, 184: 1975-1985

Liu B, Matsuoka H, Terano M. Macromol Rapid Commun, 2001, 22: 1-24

Fan Z, Feng LX, Yang SL. J Polym Sci A Polym Chem, 1996, 34: 3329-3335

Zhang L, Fu Z, Fan Z. Macromol Res, 2010, 18: 695-700

Fan Z, Zhang L, Xia S, Fu Z. J Mol Catal A-Chem, 2011, 351: 93-99

Yang H, Zhang L, Zang D, Fu Z, Fan Z. Catal Commun, 2015, 62: 104-106

Xia S, Fu Z, Liu X, Fan Z. Chin J Polym Sci, 2013, 31: 110-121

Shen X, Hu J, Fu Z, Lou J, Fan Z. Catal Commun, 2013, 30: 66-69

Kissin YV. J Catal, 2001, 200: 232-240

Rodriguez LAM, van Looy HM. J Polym Sci A-1 Polym Chem, 1966, 4: 1971-1992

Boor J Jr. Ziegler-Natta Catalysts and Polymerizations. New York: Academic Press, 1979. 403-405

Kohara T, Shinoyama M, Doi Y, Keii T. Makromol Chem, 1979, 180: 2139-2151

Doi Y. Makromol Chem Rapid Commun, 1982, 3: 635-641

Xu J, Feng L, Yang S. Macromolecules, 1997, 30: 2539-2541

Corradini P, Busico V, Cavallo L, Guerra G, Vacatello M, Venditto V. J Mol Catal, 1992, 74: 433-442

Busico V, Cipullo R, Monaco G, Vacatello M, Segre AL. Macromolecules, 1997, 30: 6251-6263

Busico V, Cipullo R, Monaco G, Talarico G, Vacatello M, Chadwick JC, Segre AL, Sudmeijer O. Macromolecules, 1999, 32: 4173-4182

Busico V, Chadwick JC, Cipullo R, Ronca S, Talarico G. Macromolecules, 2004, 37: 7437-7443

Liu B, Nitta T, Nakatani H, Terano M. Macromol Chem Phys, 2002, 203: 2412-2421

Liu B, Nitta T, Nakatani H, Terano M. Macromol Chem Phys, 2003, 204: 395-402

Liu B, Nitta T, Nakatani H, Terano M. Macromol Symp, 2004, 213: 7-18

Cossee P. J Catal, 1964, 3: 80-88

Arlman E. J Catal, 1964, 3: 89-98

Arlman E, Cossee P. J Catal, 1964, 3: 99-104

Seth M, Margl PM, Ziegler T. Macromolecules, 2002, 35: 7815-7829

Fan ZQ, Yu Y. Encyclop Polym Sci Technol, 2018, 1-24

Boor J Jr. Ziegler-Natta Catalysts and Polymerization. London: Acadenic Press, Inc., 1978. 244-246

Blaakmeer ESM, Antinucci G, van Eck ERH, Kentgens APM. J Phys Chem C, 2018, 122: 17865-17881

Guzeev B, Mladentsev D, Sharikov M, Goryunov G, Uborsky D, Voskoboynikov A. Synthesis, 2019, 51: 1399-1407

Breuza E, Antinucci G, Budzelaar PHM, Busico V, Correa A, Ehm C. J Phys Chem C, 2018, 122: 9046-9053

Blaakmeer ESM, Antinucci G, Correa A, Busico V, van Eck ERH, Kentgens APM. J Phys Chem C, 2018, 122: 5525-5536

Ratanasak M, Parasuk V. RSC Adv, 2016, 6: 112776-112783

Busico V, Corradini P, De Martino L, Proto A, Albizzati E. Makromol Chem, 1986, 187: 1115-1124

Noristi L, Barbè PC, Baruzzi G. Makromol Chem, 1991, 192: 1115-1127

Sacchi MC, Tritto I, Shan C, Mendichi R, Noristi L. Macromolecules, 1991, 24: 6823-6826

Sacchi MC, Forlini F, Tritto I, Mendichi R, Zannoni G, Noristi L. Macromolecules, 1992, 25: 5914-5918

Kioka M, Kashiwa N. J Mol Catal, 1993, 82: 11-16

Kakugo M, Miyatake T, Naito Y, Mizunuma K. Macromolecules, 1988, 21: 314-319

Shen X, Fu Z, Hu J, Wang Q, Fan Z. J Phys Chem C, 2013, 117: 15174-15182

Vittoria A, Meppelder A, Friederichs N, Busico V, Cipullo R. ACS Catal, 2017, 7: 4509-4518

Pino P, Giannini U, Porri L. Encyclopedia of Polymer Science and Engineering. 2nd ed. New York: Wiley, 1987. 147-220

Cossee P. Stereochemistry of Macromolecules. Ketley AD, ed. New York: Marcel Dekker, 1967. 145-175

Arlman E. J Catal, 1966, 5: 178-189

Taube R, Gehrke JP, Radeglia R. J Organomet Chem, 1985, 291: 101-115

Taube R, Schmidt U, Gehrke JP, Böhme P, Langlotz J, Wache S. Makromolekul Chem Macromol Sympo, 1993, 66: 245-260 
96 Sieler J, Kempe R, Wache S, Taube R. J Organomet Chem, 1993, 455: 241-246

97 Tobisch S. Acc Chem Res, 2002, 35: 96-104

98 Tobisch S. Organometallics, 2003, 22: 2729-2740

99 Tobisch S, Taube R. Organometallics, 1999, 18: 3045-3060

100 Feng Y, Song J, Huang B. China Syn Rubber Indust, 1999, 22: 276-280 (in Chinese) [冯玉红, 宋景社, 黄宝琛. 合成橡胶工业, 1999, 22: 276280]

101 Song JS, Huang BC, Yu DS. J Appl Polym Sci, 2001, 82: 81-89

102 He A, Huang B, Jiao S, Hu Y. J Appl Polym Sci, 2003, 89: 1800-1807

103 He A, Yao W, Jia Z, Huang B, Jiao S. Acta Polym Sin, 2002, 1: 19-24 (in Chinese) [贺爱华, 姚薇, 贾志峰, 黄宝琛, 焦书科. 高分子学报, 2002, 1: 19-24]

104 He A, Yao W, Huang B, Huang Y, Jiao S. J Appl Polym Sci, 2004, 92: 2941-2948

105 Zhang X, Cui H, Song L, Ren H, Wang R, He A. Compos Sci Tech, 2018, 158: 156-163

106 Wang H, Zhang J, Ma Y, Wang R, He A. Chem J Chin Univ, 2017, 38: 2095-2101 (in Chinese) [王浩, 张剑平, 马韵升, 王日国, 贺爱华. 高等 学校化学学报, 2017, 38: 2095-2101]

107 He A, Yao W, Huang G, Huang B, Jiao S, Hu Y. China Elastom, 2002, 12: 1-6 (in Chinese) [贺爱华, 姚薇, 黄光兴, 黄宝琛, 焦书科, 胡友良. 弹性体, 2002, 12: 1-6]

108 Peng J, Yao W, Du K, Huang B. China Elastom, 2003, 13: 1-4 (in Chinese) [彭杰, 姚薇, 杜凯, 黄宝琛. 弹性体, 2003, 13: 1-4]

109 He A, Yao W, Jia Z, Huang B, Jiao S. China Syn Rubber Indust, 2002, 25: 75-79 (in Chinese) [贺爱华, 姚薇, 贾志峰, 黄宝琛, 焦书科. 合成橡 胶工业, 2002, 25: 75-79]

110 Zhang Q, Jiang X, He A. Chin J Polym Sci, 2014, 32: 1068-1076

111 He A, Yao W, Huang B, Jiao S. Acta Chim Sin, 2001, 59: 1793-1797 (in Chinese) [贺爱华, 姚薇, 黄宝琛, 焦书科. 化学学报, 2001, 59: 17931797]

112 Jiang X, Zhang Q, He A. Chin J Polym Sci, 2015, 33: 815-822

113 Du K, Yao W, Guo C, He A, Huang B. China Syn Rubber Indust, 2011, 34: 29 -33 (in Chinese) [杜凯, 姚薇, 郭常青, 贺爱华, 黄宝琛. 合成橡胶 工业, 2011, 34: 29-33]

114 He A, Yao W, Guo C, He A, Huang B. China Syn Rubber Indust, 2011, 34: 186-189 (in Chinese) [贺爱华, 姚薇, 郭常青, 贺爱华, 黄宝琛. 合成 橡胶工业, 2011, 34: 186-189]

115 Du K, Peng J, Yao W, Li X, Zhao Y, Huang B. Rubber Indust, 2005, 52: 716-718 (in Chinese) [杜凯, 彭杰, 姚微, 李旭东, 赵永仙, 黄宝琛. 橡 胶工业, 2005, 52: 716-718]

116 Liu X, Li W, Niu Q, Wang R, He A. Polymer, 2018, 140: 255-268

117 Niu Q, Li W, Liu X, Wang R, He A. Polymer, 2018, 143: 173-183 


\title{
Progress on coordination polymerization mechanism of olefins catalyzed by heterogeneous $\mathrm{TiCl}_{4} / \mathrm{MgCl}_{2}$ type Ziegler-Natta catalyst
}

\author{
Qingtao Niu, Wei Peng, Aihua He* \\ Shandong Provincial Key Laboratory of Olefin Catalysis and Polymerization, Key Laboratory of Rubber-Plastics (Ministry of Education), School of \\ Polymer Science and Engineering, Qingdao University of Science and Technology, Qingdao 266042, China \\ *Corresponding authors (email: ahhe@qust.edu.cn; aihuahe@iccas.ac.cn)
}

\begin{abstract}
Heterogeneous $\mathrm{TiCl}_{4} / \mathrm{MgCl}_{2}$ type Ziegler-Natta catalysts (supported type Ziegler-Natta catalysts) have been the most important industrial catalysts in polyolefin field due to the high polymerization activity, high stereoselectivity and low preparation cost. This paper reviewed the recent progress on the coordination polymerization mechanism of $\alpha$ olefins (ethylene, propylene) and conjugated dienes (isoprene, butadiene) including the absorption of $\mathrm{TiCl}_{4}$ on $\mathrm{MgCl}_{2}$ surface, the alkylation and reduction of Ti, the number, valence, structure, model of active centers, the effect of electron donor and alkylaluminum on catalytic mechanism etc. The opportunities and challenges of olefins polymerization catalysed by supported type Ziegler-Natta catalysts were proposed.
\end{abstract}

Keywords: heterogeneous, supported type Ziegler-Natta catalyst, $\alpha$-olefin, conjugated diene, polymerization mechanism

doi: $10.1360 /$ SSC-2019-0037 